\title{
Uma discussão necessária sobre a vulnerabilidade do consumidor: avanços, lacunas e novas perspectivas
}

\author{
Rosana Oliveira da SiLVA ${ }^{1}$ \\ DENISE FRANCA BARROS ${ }^{1}$ \\ TÂNIA Maria de Oliveira Almeida Gouveia ${ }^{2}$ \\ Daniel de Oliveira Barata Merabet ${ }^{1}$ \\ ${ }^{1}$ UNIVERSIDADE DO GRANDE RIO (UNIGRANRIO), RIO DE JANEIRO - RJ, BRASIL \\ 2 UNIVERSIDADE DO ESTADO DO RIO DE JANEIRO (UERJ), RIO DE JANEIRO - RJ, BRASIL
}

\begin{abstract}
Resumo
A pesquisa sobre o consumidor não se ocupa comumente dos problemas causados e/ou agravados pelas atividades de mercado. Entretanto, problemas econômicos, sociais, ambientais e de saúde pública podem ser atribuídos a determinadas práticas de mercado, nocivas a distintos agentes que nele atuam. Assim, este artigo tem o objetivo de examinar como o conceito de vulnerabilidade do consumidor vem sendo discutido na literatura de marketing e de consumo, por meio de uma revisão da literatura dos últimos 25 anos na produção nacional e internacional de congressos científicos e periódicos. Procura-se identificar as diferentes perspectivas e definições nas principais fontes em que a temática é discutida, com o objetivo de sugerir uma definição abrangente para a vulnerabilidade na área de marketing e que leva em conta a vulnerabilidade de outros agentes. Identificamos que a vulnerabilidade é um tema que vem adquirindo importância, apesar da predominância de uma perspectiva gerencialista, que prioriza a geração de conhecimento benéfico às empresas. Parece-nos urgente que Marketing e Estudos de Consumo interessem-se por conhecimento que proteja e beneficie o consumidor e a sociedade em geral. Acreditamos que o estudo da vulnerabilidade também deve suscitar o desenvolvimento de políticas públicas adequadas e a discussão sobre as atividades reguladoras, para além do consumidor. Assim, com base nos resultados, sugerimos que a vulnerabilidade pode ser definida como um estado de fragilidade de indivíduos frente às práticas de mercado e que pode manifestar-se em diferentes etapas no processo de produção, comercialização e consumo.
\end{abstract}

Palavras-chave: Vulnerabilidade do consumidor. Sistemas de marketing. Estudos de consumo. Políticas públicas. Macromarketing.

\section{A necessary discussion on consumer vulnerability: advances, gaps, and new perspectives}

\begin{abstract}
Consumer research is not usually concerned with problems caused or aggravated by market activities. However, economic, social, environmental, and public health problems can be attributed to certain market practices, which are harmful to different market agents. This article examines how consumer vulnerability has been discussed in the marketing and consumer literature through a national and international literature review of the last 25 years. It seeks to identify the different perspectives and definitions in researches that discuss the topic and suggests a comprehensive definition of vulnerability in the marketing area, which considers the vulnerability of other agents. We identified vulnerability as an issue gaining importance, despite the predominance of a managerialist perspective, which emphasizes the generation of knowledge that benefits companies. It seems urgent that marketing and consumer studies are interested in knowledge that protects and benefits consumers and society in general. We believe that the study of vulnerability should also encourage the development of adequate public policies and the discussion of regulatory activities, in addition to the consumer. Thus, from the results, we suggest that vulnerability can be defined as a state of individuals' fragility in the face of market practices, which can manifest in different stages in production, commercialization, and consumption processes.
\end{abstract}

Keywords: Consumer vulnerability. Market systems. Consumer studies. Public policy. Macromarketing.

\section{“Una discusión necesaria sobre la vulnerabilidad del consumidor: avances, brechas y nuevas perspectivas}

\begin{abstract}
Resumen
La investigación sobre el consumidor no se ocupa comúnmente de los problemas causados y/o agravados por las actividades del mercado. Sin embargo, hay problemas económicos, sociales, ambientales y de salud pública que pueden atribuirse a ciertas prácticas de mercado, perjudiciales para los diferentes agentes que operan en él. Por lo tanto, este artículo tiene como objetivo examinar cómo se ha discutido el concepto de vulnerabilidad del consumidor en la literatura de marketing y consumo, a través de una revisión de la literatura de los últimos 25 años en la producción nacional e internacional de congresos científicos y periódicos. Busca identificar las diferentes perspectivas y definiciones, en las principales fuentes en las que se discute el tema, para sugerir una definición integral de vulnerabilidad en el área de marketing, que considera la vulnerabilidad de otros agentes. Identificamos que la vulnerabilidad es un tema que ha ido ganando importancia, a pesar del predominio de una perspectiva gerencial, que prioriza la generación de conocimiento que beneficia a las empresas. Parece urgente que los estudios de marketing y consumo se interesen en un conocimiento que proteja y beneficie al consumidor y a la sociedad en general. Consideramos que el estudio de la vulnerabilidad también debería alentar el desarrollo de políticas públicas adecuadas y la discusión de actividades reguladoras, más allá del consumidor. Por lo tanto, a partir de los resultados, sugerimos que la vulnerabilidad se puede definir como un estado de fragilidad de los individuos frente a las prácticas de mercado, que puede manifestarse en diferentes etapas en el proceso de producción, comercialización y consumo.
\end{abstract}

Palabras clave: Vulnerabilidad del consumidor. Sistemas de marketing. Estudios de consumo. Políticas públicas. Macromarketing. 


\section{INTRODUÇÃo}

A pesquisa sobre o consumidor não se ocupa comumente dos problemas causados e/ou agravados pelas atividades de mercado (Sauerbronn, 2013; Shultz II \& Holbrook, 2009), mas problemas econômicos, sociais, ambientais e de saúde pública podem ser atribuídos a determinadas práticas de mercado. As mesmas práticas de produção, comercialização e consumo de produtos e serviços que podem gerar empregos, desenvolvimento econômico, bem-estar, entre outros resultados positivos, também podem produzir resultados negativos diretos e/ou indiretos, cujo alcance não é limitado aos consumidores, podendo atingir distintos agentes do mercado e a sociedade em geral (Nason, 1989; Shultz II \& Holbrook, 2009).

Os profissionais de marketing são, por vezes, acusados de explorar consumidores, especialmente aqueles em posição de vulnerabilidade (Hackley, 2009; Shultz II \& Holbrook, 2009). Smith e Cooper-Martin (1997) e Karpatkin (1999) apontaram que várias empresas beneficiaram-se com práticas de segmentação antiéticas, por ter como objetivo de mercado populações sabidamente vulneráveis, vendendo produtos reconhecidamente nocivos como tabaco, álcool, fast-food, criando e/ou reforçando comportamentos de adição e problemas de saúde. Além disso, as consequências das atividades de marketing e/ou do consumo de produtos podem atingir populações de forma diferente, o que sugere a existência de indivíduos e grupos que podem ser mais vulneráveis a determinados problemas e/ou ações da indústria (Commuri \& Ekici, 2008).

Para além do comportamento antiético que empresas e/ou profissionais de marketing podem assumir ao criar, divulgar e comercializar seus produtos e serviços e, portanto, prejudicar seus consumidores, a literatura de marketing e consumo deve voltar-se a problemas que são consequências de atividades mercadológicas cotidianas. Nason (1989) aponta que a produção e o consumo de produtos e serviços podem causar efeitos nocivos aos indivíduos e às organizações - mesmo que de forma não premeditada e intencional. Problemas ambientais gerados pelo descarte de substâncias usadas nos processos produtivos, doenças causadas pelo manuseio de agrotóxicos, estigmas sofridos por famílias de alcoólatras são alguns exemplos de vicissitudes que podem atingir trabalhadores, fornecedores de indústrias, consumidores e a sociedade em geral. Diante desses e de outros problemas, faz-se necessário o envolvimento de entes reguladores e desenvolvimento de políticas públicas.

À medida que tais consequências decorrentes das atividades de mercado acumulam-se, geram complicações (in)diretas agregadas, capazes de influenciar a saúde pública (como no caso de doenças associadas ao tabaco, que sobrecarregam o orçamento público de hospitais), a saúde do trabalhador (como aqueles expostos aos defensivos agrícolas) ou o meio ambiente, cujos danos são potencialmente mais amplos, uma vez que incluem os anteriores.

É possível observar, em todos os exemplos, que existe algum grau de vulnerabilidade - esta pode estar limitada ao consumidor ou afetar diferentes agentes. No entanto, os problemas decorrentes das atividades mercadológicas não são comumente atribuídos à disciplina e/ou prática do marketing. Essa pode ser até mesmo a razão pela qual a vulnerabilidade em marketing ficou restrita ao consumidor, haja vista os termos "consumidores vulneráveis" e "vulnerabilidade do consumidor". Normalmente, os problemas decorrentes de atividades de mercado são vistos como um erro de gestão e não como parte da própria estrutura mercadológica. Argumentamos aqui que, ao não reconhecer tais problemas (ao menos em parte) como consequência das atividades de produção, comercialização e consumo, tanto a academia quanto os praticantes de marketing negligenciam aspectos importantes de seu escopo e de sua relação com a sociedade.

As relações entre mercado, as práticas de marketing e a sociedade eram tema importante nos momentos iniciais da disciplina de marketing (Boschi, Barros \& Sauerbronn, 2016; Nason, 2011; Shapiro, Tadajewski \& Shultz II, 2009; Wilkie \& Moore, 2003). Procurava-se responder de que forma as práticas de marketing poderiam gerar benefícios e/ou problemas para a sociedade e se tais comportamentos deveriam ou não ser regulados (Nason, 2011; Wilkie \& Moore, 2003). Tal perspectiva perdeu relevância com a emergência do paradigma gerencial de marketing no final da década de 1960 (Hunt, 1976; Layton \& Grossbart, 2006), de concepção mais restrita, caracterizando-se por enfatizar as dimensões lucro, micro e normativa (Hunt, 1976).

No Brasil, o conceito de vulnerabilidade do consumidor (doravante VC) ganha importância originalmente no Direito. Até por esse motivo, na década de 70 falava-se em defesa do consumidor (Bitencourt, 2004). Apesar de na década de 60 já haver discussão na área de marketing sobre a assimetria entre grandes empresas e consumidores (Hemais \& Faria, 2012), o mesmo não ocorria no Brasil. A VC somente ganha espaço, no país, na década de 90 (Baker, Gentry \& Rittenburg, 2005). 
No entanto, questões sociais são ignoradas pela grande maioria dos pesquisadores de marketing (Sheth \& Sisodia, 2005). Ao marginalizar assuntos como regulação e consequências das atividades mercadológicas, o bem-estar do consumidor e da sociedade em geral pode ficar comprometido, especialmente quando não se considera que estes estão em situação fragilizada.

Advogamos que o tema merece mais atenção e intentamos contribuir para uma chamada à ação ao fornecer uma visão geral que sintetiza e analisa a produção sobre um tema reconhecidamente tratado de forma fragmentada (Pavia \& Mason, 2014; Piacentini, Dunnett \& Hamilton, 2014), em que se desconsidera a vulnerabilidade de outros agentes. Sendo assim, objetivamos examinar neste artigo como o conceito de VC tem sido discutido na literatura de marketing e de consumo por meio de uma revisão de literatura dos últimos 25 anos na produção nacional e internacional, identificando as diferentes perspectivas e definições nas principais fontes em que a temática é discutida, visando sugerir uma definição abrangente para a vulnerabilidade na área de marketing, que considera também a vulnerabilidade de outros agentes presentes no sistema.

\section{Vulnerabilidade do Consumidor: Considerações Iniciais}

Vulnerabilidade sugere uma condição desfavorável, algum nível de fragilidade que expõe o indivíduo a potencial perigo, colocando-o em inferioridade. No Direito, o conceito de vulnerável é particularmente importante para que possam ser garantidos direitos iguais para todo e qualquer indivíduo que precise de proteção diferenciada (Oliveira, 2010).

A preocupação com a proteção das relações de consumo, complexas e conflituosas, surge, no Direito, no final do século XIX, na época do aparecimento da produção em série, o que, mais tarde, levou à criação de organizações que tinham como premissa defender os consumidores, como a Proteção e Defesa do Consumidor (PROCON) e o Instituto Brasileiro de Defesa do Consumidor (IDEC). No Código de Defesa do Consumidor, a Lei no 8.078 (1990) visa proteger o consumidor de situações de vulnerabilidade diante da ação das empresas.

Bitencourt (2004) defende que todo consumidor é vulnerável nas relações com os fornecedores de bens e serviços, ficando à mercê daqueles que têm o controle empresarial. A necessidade de proteção pode ser ainda maior para pessoas de baixa renda, que ainda têm de lidar com a discriminação (Santo \& Hemais, 2017). Ferrari e Takey (2014, p. 6) explicam que "[...] a vulnerabilidade jurídica emana das dificuldades que os consumidores encontram para defender os seus direitos junto aos fornecedores, uma vez que esses impõem muitas dificuldades". Sob a perspectiva jurídica, a vulnerabilidade está relacionada ao "[...] lado fraco de um assunto ou questão [pessoas físicas e jurídicas] [...]" e ao "[...] ponto por onde alguém pode ser atacado ou ofendido [permanente ou temporariamente]" (Lima, 2011, p. 245), sem relação com características socioeconômicas e culturais.

Embora o primeiro trabalho sobre a VC, em 1963, seja o livro The poor pay more: consumer practices of low-income families, de David Caplovitz (Baker et al., 2005), somente na década de 1990 houve avanço nas pesquisas em Marketing e Consumo. Tais estudos não trazem definição clara, mas replicam como tribunais definem VC (Baker et al., 2005): "[...] a conceituação da vulnerabilidade é importante porque envolve o bem-estar do consumidor, bem como as respostas das empresas, políticas e da sociedade ao mal-estar do consumidor" (Baker, Geiger-Oneto \& Gentry, 2011, p. 176). O que há em comum entre tais pesquisas é a frequente relação entre vulnerabilidade e desvantagem, embora nem todos aqueles em desvantagem experimentem vulnerabilidades e pessoas sem desvantagens, também percebidas, possam experimentar a vulnerabilidade (Baker et al., 2005).

$\mathrm{O}$ artigo mais citado sobre o tema, define a VC como:

[...] um estado de impotência que resulta de um desequilíbrio nas interações de mercado ou a partir do consumo de mensagens de marketing e produtos. [...] ela ocorre quando o controle não está nas mãos de um indivíduo, criando uma dependência de fatores externos (por exemplo, comerciantes) para criar a equidade no mercado (Baker et al., 2005, p. 7).

De forma complementar, para Visconti (2016, p. 371) é “[...] uma condição de mercado que expõe um ou mais indivíduos ao risco de obter utilidade limitada das transações de mercado, com implicações para seu bem-estar". A VC pode diferir em termo de etiologia (e.g., cultural, econômica, social), duração (permanente ou transitória), locus (mercado versus vida social), significado (consumista versus não consumista) e número de pessoas que compartilham a condição de vulnerável (individual ou social). Portanto o autor amplia a concepção da vulnerabilidade, e reconhece que existem classes mais suscetíveis de danos (e.g., imigrantes, sem-teto e gays) (Visconti, 2016, p. 372). 
Baker e Mason (2012) reconhecem a falta de pesquisas mais abrangentes sobre a vulnerabilidade, porque mercado e consumo podem ser simultaneamente fonte de conflitos, riscos, vulnerabilidade, significado e prazer que afetam diversos públicos. A edição especial do Journal of Macromarketing, em 2005, destacou lacunas e a necessidade de ampliação de pesquisas (Commuri \& Ekici, 2008) bem como o papel dos acadêmicos de macromarketing na compreensão da VC (Rittenbourg \& Lunde, 2016). Nesse caso, o macromarketing é entendido como o estudo das relações sistêmicas entre os atores de marketing (Bagozzi, 1977) ou como o estudo sobre o sistema de comercialização e os mútuos impactos e consequências entre tal sistema e a sociedade (Hunt \& Burnett, 1982), além estar relacionado à preocupação com o bem-estar social (Miranda \& Arruda, 2004). A Transformative Consumer Research (TCR) também é uma área por meio da qual a VC costuma ser tratada, certamente em virtude de essa área dedicar-se "[...] a estudar e compreender o comportamento de consumo com vistas ao bem-estar individual e coletivo, oferecendo à sociedade uma resposta positiva aos impactos negligentes do marketing" (Pinto, Batinga, Ássimos \& Almeida, 2016, p. 54). Assim, notadamente, as áreas de Macromarketing e TCR concentram a maior parte da incipiente pesquisa sobre VC.

\section{PROCEDIMENTOS METODOLÓGICOS}

A exemplo do clássico artigo de Arnould e Thompson (2005) e das recentes pesquisas de Coda e Castro (2019), Demo, Fogaça e Costa (2018) e Kuzma, Doliveira e Silva (2017), realizamos uma revisão da literatura para identificar como o conceito de VC é tratado. Para Ferenhof e Fernandes (2016, p. 551), a revisão de literatura é "[...] a base para a identificação do atual conhecimento científico" e de "hiatos a serem explorados em determinados assuntos" por meio de "[...] metodologia [que] consiste em uma série de procedimentos preestabelecidos" (Coda \& Castro, 2019, p. 260).

Importante destacar que, por se tratar de tema emergente - mesmo não sendo novo -, incluímos artigos de congressos, uma vez que esses espaços são destinados à discussão de temas em evolução. Assim, analisamos os últimos 25 anos da produção nacional de congressos científicos (EMA, EnANPAD, SemeAd, AdCONT e ENEC) e periódicos (extraídos dos indexadores SCIELO Brasil e SPELL) e internacional (Annals of Macromarketing Conference, Journal of Macromarketing, Journal of Public Policy e Journal Public Policy \& Marketing, Journal of Business Ethics e Journal of Marketing). Os congressos escolhidos são específicos ou tem tracks de Marketing, bem como são eventos longevos (de 8 a 44 anos). 0 Macromarketing Conference possui espaço exclusivo para artigos sobre VC. Selecionamos os periódicos internacionais de marketing, macromarketing, ética e políticas públicas (eixos onde a vulnerabilidade é ou pode ser tratada) de notável relevância, pela longevidade e classificação no sistema Qualis-Capes como A1 e/ou com índice JCR ou H-index. Quanto aos nacionais, optamos pelos dois indexadores e artigos classificados pela Capes como Qualis A2 e B2 (com exceção da Revista Interdisciplinar de Marketing, classificada como B4). As classificações dos periódicos foram verificadas na Plataforma Sucupira (2018).

Utilizamos como termos-chave para a pesquisa "vulnerabilidade" e "vulnerável" ou "vulnerability" e "vulnerable". Apesar do grande número de resultados (mais de 1.500 artigos), a leitura inicial dos resumos revelou que a grande maioria não tratava da VC, o que levou ao descarte de tais artigos, além daqueles em duplicidade e/ou de outras áreas, restando 29 nacionais e 138 internacionais.

Concentramo-nos nos aspectos teóricos e empíricos, na análise temática dos enquadramentos teóricos, objetivos, conclusões e resultados, de forma semelhante ao estudo de Coda e Castro (2019), e na avaliação crítica dos artigos, tal como realizado por Kuzma et al. (2017). Nossa análise temática revelou três grandes perspectivas a respeito da vulnerabilidade, conforme descrito no Quadro 1. 
Quadro 1

Perspectivas sobre a Vulnerabilidade

\begin{tabular}{|c|c|c|c|}
\hline Perspectivas & $\begin{array}{c}\text { Fatores que Causam } \\
\text { a Vulnerabilidade }\end{array}$ & $\begin{array}{l}\text { Exemplos ou } \\
\text { Explicações }\end{array}$ & Exemplo \\
\hline \multirow{3}{*}{$\begin{array}{l}\text { Condições } \\
\text { que levam à } \\
\text { vulnerabilidade }\end{array}$} & $\begin{array}{l}\text { Características } \\
\text { individuais }\end{array}$ & $\begin{array}{l}\text { Pobres, crianças, minorias étnicas, imigrantes, } \\
\text { mulheres, idosos, pessoas com deficiência (PCD). }\end{array}$ & \multirow{3}{*}{$\begin{array}{l}\text { Baker et al. } \\
\text { (2005) }\end{array}$} \\
\hline & Estado transitório & Casamento, morte e divórcio. & \\
\hline & Fatores externos & $\begin{array}{l}\text { Estigmatização, discriminação, } \\
\text { elementos físicos e logísticos. }\end{array}$ & \\
\hline $\begin{array}{l}\text { Questões } \\
\text { éticas }\end{array}$ & $\begin{array}{l}\text { Estratégias de } \\
\text { marketing podem } \\
\text { influenciar a } \\
\text { vulnerabilidade }\end{array}$ & $\begin{array}{c}\text { Segmentação de populaçã o vulnerável, } \\
\text { venda de produtos nocivos e responsabilidade } \\
\text { moral do marketing. }\end{array}$ & $\begin{array}{c}\text { Smith e } \\
\text { Cooper-Martin } \\
(1997)\end{array}$ \\
\hline $\begin{array}{l}\text { VC como resultado } \\
\text { dos sistemas } \\
\text { de marketing }\end{array}$ & $\begin{array}{l}\text { Atividades do } \\
\text { mercado podem } \\
\text { gerar consequências } \\
\text { negativas }\end{array}$ & $\begin{array}{c}\text { Práticas de produção, comercialização e consumo } \\
\text { de produtos e serviços podem produzir resultados } \\
\text { negativos di retos e/ou indiretos sobre seus } \\
\text { consumidores e/ou sobre a sociedade em geral. }\end{array}$ & $\begin{array}{l}\text { Nason (1989) } \\
\text { e Shultz II e } \\
\text { Holbrook (2009) }\end{array}$ \\
\hline
\end{tabular}

Fonte: Elaborado pelos autores.

É importante ressaltar que as perspectivas são diferentes entre si, mas podem estar presentes de forma simultânea em alguns trabalhos. Por tal motivo, algumas pesquisas constam em mais de uma das supracitadas perspectivas. Dessa forma, observamos que a VC é tratada na literatura sob diferentes perspectivas, mas que possuem certa complementaridade entre si.

\section{ANÁLISE DOS RESULTADOS}

\section{Condições que levam à vulnerabilidade}

Esta perspectiva diz respeito ao conjunto de trabalhos que discutem a vulnerabilidade em termos de seus antecedentes, de forma a criar definições úteis para entender como tratá-la. A permanência ou transitoriedade da vulnerabilidade é importante nesta categoria.

A vulnerabilidade é comumente vista como condição transitória, algo que ocorre em momentos difíceis (luto, divórcio...) (Commuri \& Ekici, 2008), desastres naturais (e.g., Baker, 2009) ou na dificuldade de tomar decisões no momento da compra (especialmente PCD, sem-teto...) (e.g., Pechmann et al., 2011). Há também estados permanentes, como nos casos de dificuldades de compreensão (e.g., Viswanathan \& Gau, 2005) causadas pelo analfabetismo funcional. Consequentemente tem-se a visão de que as estratégias de marketing são eticamente questionáveis ou equivocadas, já que pressupõem consumidores com conhecimento pleno. A alfabetização do consumidor inclui a agência do consumidor, o conhecimento e a capacidade de gerenciar suas escolhas, independentemente de questões sociais ou situacionais, de "forma a satisfazer suas necessidades no mercado" (Adkins \& Ozanne, 2005, p. 93). Mansfield e Pinto (2008) investigaram PCD cognitiva e o conhecimento básico de cartão de crédito, concluindo que eles têm um potencial grande de sofrerem danos - econômicos, físicos e psicológicos. Mason e Pavia (2014) sugerem relação entre vulnerabilidade e corpo, seja ele saudável ou com alguma condição de doença, idade avançada ou deficiência - indicando um terreno fértil de pesquisa para questões relacionadas ao corpo como condutor fundamental, que limita ou não a capacidade de engajamento nas relações de mercado.

Smith e Cooper-Martin (1997, p. 4) definem como consumidores vulneráveis: "[...] aqueles mais suscetíveis a danos econômicos, físicos ou psicológicos, ou como um resultado de transações econômicas devido a características que limitam a sua capacidade de maximizar a sua utilidade e bem-estar". Tal "consumidor em situação de risco" devido a deficiências ou circunstâncias pessoais, "[...] pode ser prejudicado por práticas dos comerciantes ou pode ser incapaz ou não quer tirar pleno partido das oportunidades de mercado" (Pechmann et al., 2011, p. 23). Inicialmente concentradas em consumidores pobres, analfabetos, minorias raciais, imigrantes ou mulheres, as pesquisas ampliam-se nos anos 2000 para PCD, jovens, crianças, sem-teto (por causa de desastres), lésbicas, gays, bissexuais e transgêneros. $O$ trabalho de Visconti (2016) permite verificar essas novas abordagens. 
Frequentemente, pensar sobre os antecedentes da vulnerabilidade exige questionar se é uma questão individual ou própria de determinados grupos de consumidores, embora se reconheça como consequência de "relações e processos sociais complexos" (Hilhorst \& Bankoff, 2004, p. 1). Para Baker et al. (2005), apesar de algumas características e fatores facilitarem a vulnerabilidade, defini-la a partir de grupos não é o melhor caminho, como pontuado por Brenkert (1998), para quem somente os consumidores que tipicamente estão sujeitos a algum nível substancial de dano são vulneráveis (embora esclareça que ser "suscetível" à vulnerabilidade não significa necessariamente ser vulnerável). Berg (2015) e Woodliffe (2007) também questionam a ideia de que determinados grupos são vulneráveis argumentando que nem todos podem ser considerados iguais pelo mercado, já que alguns possuem mais dificuldades do que outros. Berg (2015, p. 284) estudou os idosos como consumidores potencialmente vulneráveis e afirma que, apesar de algumas limitações físicas, parece ser menos provável que, em relação a outros grupos etários, tomem decisões infelizes no momento de consumo.

Várias pesquisas abordam como idade, raça, sexo, escolaridade e renda podem influenciar a capacidade de o consumidor entender e tomar decisões, apontando as características demográficas como aquelas que podem colocá-lo em desvantagem (Hill, 2005). Shultz II e Holbrook (2009) usam educação e capital cultural e renda para criar uma tipologia: vulneráveis economicamente (não têm acesso a meios benéficos), vulneráveis culturalmente (não têm conhecimento dos benefícios), duplamente vulneráveis (não têm acesso a meios benéficos e conhecimento dos benefícios) e os invulneráveis (têm acesso e conhecimento). Diferentemente, Adkins e Jae (2010) mencionam a importância de identificar grupos mais suscetíveis, entendendo que, para alguns, a vulnerabilidade pode ser permanente - conforme apontam Commuri e Ekici (2008).

Para Diniz, Pereira e Bellini (2014), a vulnerabilidade está relacionada a questões de fragilidade do consumidor e pode ser situacional e circunstancial, não sendo diretamente ligada ao nível econômico-social dos indivíduos - como no caso dos jovens viciados em internet. No que se refere aos jovens, há indícios de uma vulnerabilidade permanente. Souza e Silva (2006) chamam atenção para a vulnerabilidade identificada nos jovens diante das estratégias de marketing, visto terem sido "educados" e "condicionados" a aspectos relacionados à sociedade de consumo, que contribuiu para que os jovens investigados se tornassem dependentes e incapazes de exigir e criticar.

Estados transitórios derivados de luto ou doenças são causas da VC (Hamilton, Dunnet \& Piacentini, 2015, p. 1) que podem ser agravados. Para Rittenburg e Lunde (2016, p. 198), apesar de os comerciantes não terem (ou pouco terem) controle sobre "as características ou estados individuais", geralmente, "têm um nível de influência sobre algumas das condições externas" e, certamente, "têm controle sobre o contexto de consumo em que a vulnerabilidade é vivenciada". Dessa forma, alguns agentes aproveitam-se da falta de conhecimento dos consumidores sobre aspectos relacionados ao mercado, bem como de estados transitórios que os tornam mais frágeis - como no luto (Silva \& Barros, 2018).

\section{Questões éticas}

Nesta perspectiva, prevalecem discussões éticas sobre a vulnerabilidade, até mesmo criticando o papel do marketing na ampliação da VC. Embora possam oferecer conforto e segurança para milhares de consumidores, "[...] os profissionais de marketing são frequentemente ridicularizados, muitas vezes com razão, por explorarem clientes em geral e por tirarem proveito dos consumidores vulneráveis, em particular" (Shultz II \& Holbrook, 2009, p. 124). Observa-se, nesse sentido, que vulnerabilidade e oportunismo caminham juntos, como também apontado por Silva e Barros (2018).

De forma complementar, Jones e Middleton (2007) afirmam que os consumidores não se mostram capazes de distinguir se seu nível de vulnerabilidade é alto ou baixo diante das estratégias de marketing. Isto é, o consumidor não consegue avaliar adequadamente se as estratégias de marketing das empresas são éticas ou não em termos de contribuição socioambiental e os danos que os produtos podem causar. Jones e Middleton (2007) ressaltam que os consumidores podem até querer consumir produtos considerados "corretos", mas muitas vezes não conseguem fazê-lo porque não entendem a "natureza do produto".

Existem também trabalhos que destacam o fato de o valor da empresa ser afetado de forma negativa diante da adoção de práticas consideradas não éticas voltadas para consumidores mais vulneráveis (Tipton, Bharadwaj \& Robertson, 2009). Wolburg (2005) pondera que, quando o alvo é vulnerável e são ofertados produtos potencialmente prejudiciais (e.g., empréstimos consignados, cigarros e álcool), essa relação torna-se incorreta. Esta visão parte do pressuposto de que a VC é uma externalidade das atividades mercadológicas, portanto não considerando-a como parte integrante da estrutura do mercado, o que é discutível e combatido por Nason (1989). 
A vulnerabilidade pode ser consequência da falta de conhecimento das empresas e da sociedade sobre os consumidores, mas também da má-fé (Silva, Abreu \& Mano, 2015), que tem potencial de dano cada vez maior "[...] com a introdução do Facebook, Twitter, e outros meios de segmentação dos consumidores" (Rittenburg \& Lunde, 2016, p. 190), incluindo manipulações psicológicas como aumento de crédito, que levaria a maiores gastos, sobretudo em públicos em estado mais suscetível à vulnerabilidade (Harrison \& Massi, 2011).

Para Smith e Cooper-Martin (1997), a preocupação maior com questões éticas surge com os produtos prejudiciais que exigem que formuladores de políticas públicas pensem em grupos potencialmente vulneráveis, para educá-los quanto aos danos que produtos podem causar. Pechmann et al. (2011, p. 23) também defendem a necessidade de políticas públicas para o que chamam de "consumidor em situação de risco" e, do mesmo modo, Shultz II e Holbrook (2009, p. 124) argumentam que "[...] precisamos desenvolver os quadros existentes de uma forma que possam inspirar novas ideias, políticas adequadas e práticas eficazes de intervenção".

\section{Vulnerabilidade do consumidor como resultado dos sistemas de marketing}

Na terceira perspectiva, encontram-se pesquisas que identificaram que o mercado concorre para a VC, pela ausência ou falhas na legislação, pela influência que exerce sobre a agência do consumidor, dentre outros exemplos. Embora bastante próxima da anterior, esta perspectiva relaciona claramente o sistema de marketing à vulnerabilidade.

O mercado de moda, por exemplo, é apontado por Ertekin e Atik (2015) e Barros, Merabet e Gouveia (2015) como uma das causas da sexualização infantil, seja retratando crianças em poses adultas e sensuais, seja fomentando o consumo de produtos e serviços não indicados para crianças e adolescentes. Pechmann, Levine, Loughlin e Leslie (2005) demonstram a vulnerabilidade dos adolescentes em relação à influência da publicidade e promoção. Silva, Barros e Gouveia (2017) discutem a popularização de suplementos alimentares esportivos de venda livre entre crianças e jovens. Isso evidencia a necessidade de regulação, pois não sendo a educação do consumidor suficiente para protegê-lo, ele se torna incapaz de compreender as tecnicalidades do produto. Silva e Barros (2018) identificaram, no mercado funerário, falhas e imperfeições, retenção e falta de informações que causam a VC.

Como destacado, frequentemente os esforços para compreensão da vulnerabilidade encontram-se no domínio do macromarketing. Por meio da abordagem deste, as consequências oriundas de atividades do mercado não devem ser consideradas externalidades, mas parte integrante das transações. As consequências das transações de mercado podem atingir diferentes grupos da sociedade de formas distintas e tal abordagem é útil para o desenvolvimento de políticas públicas necessárias voltadas ao bem-estar social (Nason, 1989), que, dentre outras questões, estejam relacionadas à VC. Shultz II e Holbrook (2009) também apontam para resultados negativos das práticas de produção, comercialização e consumo de produtos e serviços. Para Layton e Grossbart (2006), por exemplo, a avaliação de riscos econômicos, físicos, ecológicos, sociais e psicológicos dos sistemas de marketing tem sido de interesse das políticas públicas por envolver, em alguns casos, questões éticas e morais.

As tipologias de Baker e Mason (2012) e Baker et al. (2005) reconhecem a contribuição do mercado para a VC, mas Hill (2005) aponta que há poucas pesquisas sobre características estruturais do mercado e sua relação com os fatores demográficos.

A agência do consumidor é um aspecto bastante questionado (e.g., Gould \& Semann, 2014; McKeage, Crosby \& Rittenburg, 2015). Isso porque por mais que se possa imaginar que o consumidor seja soberano e tenha o poder de decisão, o mercado pode ter influência sobre a agência do consumidor, principalmente quando parte de grupo com um potencial de vulnerabilidade e/ou seja estigmatizado pela sociedade. McKeage et al. (2015, p. 71) pesquisaram uma variedade de identidades de gênero, incluindo transgênero e genderqueer, e afirmam que "[...] a influência que o mercado exerce sobre a agência do consumidor nesse contexto tem consequências potencialmente significativas para esse grupo de indivíduos". Nesse sentido, muitos autores (e.g., Mirosa \& Wooliscroft, 2013; Silva \& Barros, 2018) entendem que o consumidor não é soberano e que o sistema de marketing contribui - mesmo que inadvertidamente - para a VC.

O equilíbrio entre a intervenção e a interferência nas atividades mercadológicas é importante. Mittelstaedt, Duke e Mittelstaedt (2009) destacam a vulnerabilidade que o sistema de marketing impõe aos cuidados com a saúde. Pearson e Liu-Thompkins $(2012$, p. 1) refletem que "[...] como o marketing direto para o consumidor de testes genéticos cresce em popularidade, há uma necessidade crescente de entender melhor as implicações éticas e políticas públicas desses produtos". Dessa forma, existiriam quatro grandes tensões: “(1) vulnerabilidade versus forças, (2) mudanças radicais versus 
marginal, (3) direcionar versus não segmentar e (4) encorajar consumidores conhecedores versus consumidores ingênuos", entendendo que o mais importante é "[...] incluir os consumidores em risco como participantes do mercado e identificar futuras direções de pesquisas" (Pechmann et al., 2011, p. 23).

Contudo Rittenburg e Lunde (2016, p. 198) apontam que “[...] os decisores de políticas públicas pisam em uma linha tênue entre proteger os consumidores em risco de experimentar a vulnerabilidade e manter a liberdade de escolha e acesso a informações e produtos no mercado". Dessa forma, para os autores, o papel dos acadêmicos de macromarketing seria "[...] obter uma compreensão mais clara deste fenômeno e encontrar soluções viáveis para seus efeitos negativos, sem menosprezar os direitos individuais" (Rittenburg \& Lunde, 2016, p. 200).

\section{CONSIDERAÇÕES FINAIS}

Inovações - frequentemente vistas como benéficas - também podem impor desafios. Avanços na área da saúde, como tratamentos de fertilização e pesquisa genética, trazem potenciais problemas, alguns dos quais sem precedentes e que poderão exigir políticas públicas (Pearson e Liu-Thompkins, 2012). Da mesma forma, avanços na nutrição, como é o caso dos suplementos alimentares, já se revelam problemáticos pela popularização, até mesmo entre crianças (Silva et al., 2017).

Seja instigada por falta de conhecimento do público ou má fé das organizações, a pesquisa sobre vulnerabilidade pode fornecer subsídios que ampliem a proteção do consumidor, para além do ponto de vista legal, que pode ser limitador. Acredita-se que o estudo da vulnerabilidade também deve suscitar o desenvolvimento de políticas públicas adequadas e a discussão sobre atividades reguladoras, que protejam todos os entes do sistema de marketing. A existência do Código de Defesa do Consumidor (CDC) e outros órgãos de proteção do consumidor indicam preocupação, mas não são suficientes, pois as atividades mercadológicas podem afetar outros agentes.

Uma parcela dos artigos analisados dedica-se a definir e contextualizar a VC (e.g., Pavia \& Mason, 2014; Piacentini et al., 2014), apontando a inexistência de visão unânime sobre o termo. Observa-se, porém, que a maior parte dos estudos a define não como uma propriedade de determinados grupos ou ambientes, mas como algo contextual e não permanente. Todavia não significa que para alguns ela não possa ser permanente diante de condições que os coloquem em desvantagem e marginalizados, como no caso dos sem-teto, gays, imigrantes ilegais (Visconti, 2016). Consumidores vulneráveis e grupos dominantes podem estar engajados no mesmo contexto, mas de forma desigual e assimétrica, daí a importância da investigação sobre o bem-estar do consumidor.

O Quadro 2 apresenta as perspectivas e as principais contribuições de tal corpo de literatura, aos moldes do que fizeram Arnould e Thompson (2005). 


\section{Perspectivas e suas Contribuições}

\begin{tabular}{|c|c|c|}
\hline & Autor(es) & Contribuições \\
\hline \multirow{4}{*}{$\begin{array}{l}\text { Condições } \\
\text { que levam à } \\
\text { vulnerabilidade }\end{array}$} & $\begin{array}{l}\text { Baker et al. } \\
\quad(2005)\end{array}$ & $\begin{array}{l}\text { Definição da VC e apresentação de um modelo que considera os fatores que } \\
\text { aumentam a possibilidade de ocorrência da vulnerabilidade, as experiências } \\
\text { de vulnerabilidade e as respostas do consumidor. }\end{array}$ \\
\hline & $\begin{array}{l}\text { Commuri e Ekici } \\
\qquad(2008)\end{array}$ & $\begin{array}{l}\text { Definição da VC como soma de dois componentes: um componente sistêmico } \\
\text { baseado em classe e um transitório baseado em estado. }\end{array}$ \\
\hline & $\begin{array}{l}\text { Shultz II e Holbrook } \\
\qquad \text { (2009) }\end{array}$ & $\begin{array}{l}\text { Criação de uma tipologia de VC: duplamente vulneráveis (não têm acesso } \\
\text { a meios benéficos e não têm conhecimento dos benefícios), vulneráveis } \\
\text { economicamente (não têm acesso a meios benéficos), vulneráveis culturalmente } \\
\text { (não têm conhecimento dos benefícios) e invulneráveis (têm acesso a meios } \\
\text { benéficos e conhecimento dos benefícios). }\end{array}$ \\
\hline & $\begin{array}{l}\text { Baker e Mason } \\
\qquad(2012)\end{array}$ & $\begin{array}{l}\text { Criação de um modelo conceitual que permite analisar a vulnerabilidade, } \\
\text { considerando pressões, eventos disparadores, tensões ideológicas, estado } \\
\text { de vulnerabilidade e resiliência, bem como a atuação de vários atores. }\end{array}$ \\
\hline Questões éticas & $\begin{array}{l}\text { Smith e Cooper-Martin } \\
\qquad \begin{array}{l}\text { (1997) } \\
\text { Jones e Middleton } \\
\text { (2007) }\end{array}\end{array}$ & $\begin{array}{l}\text { Preocupação com questões relacionadas à ética nas estratégias de marketing } \\
\text { e mercado, principalmente quando se trata de consumidores vulneráveis. }\end{array}$ \\
\hline \multirow{4}{*}{$\begin{array}{l}\text { VC como resultado } \\
\text { dos sistemas de } \\
\text { marketing }\end{array}$} & $\begin{array}{l}\text { Shultz II e Holbrook } \\
\text { (2009) }\end{array}$ & $\begin{array}{l}\text { Constatação de que práticas de produção, comercialização e consumo de } \\
\text { produtos e serviços podem produzir resultados negativos diretos e/ou } \\
\text { indiretos sobre seus consumidores e/ou a sociedade em geral. }\end{array}$ \\
\hline & $\begin{array}{l}\text { Mirosa e Wooliscroft } \\
\qquad(2013)\end{array}$ & $\begin{array}{l}\text { Identificação de que os compradores são vulneráveis às forças de exploração } \\
\text { dos principais atores neoliberais globais do mercado. }\end{array}$ \\
\hline & $\begin{array}{l}\text { McKeage, Crosby e } \\
\text { Rittenburg (2015) }\end{array}$ & $\begin{array}{l}\text { Constatação de que o mercado pode facilitar ou impedir o controle e que a } \\
\text { influência do mercado sobre a agência dos consumidores tem consequências. }\end{array}$ \\
\hline & $\begin{array}{l}\text { Silva e Barros } \\
\qquad(2018)\end{array}$ & $\begin{array}{l}\text { Identificação de que as práticas do mercado influenciam e aprofundam a } \\
\text { vulnerabilidade e de que o consumidor não tem agência (passivos). }\end{array}$ \\
\hline
\end{tabular}

Fonte: Elaborado pelos autores com base nos dados de pesquisa.

Nossa análise revela que as pesquisas sobre VC já formam um importante corpo teórico, o qual ainda merece atenção. Propomos, por meio de tal revisão, uma definição do conceito de vulnerabilidade que pode orientar potenciais pesquisas empíricas: a vulnerabilidade é um estado de fragilidade de indivíduos frente às práticas de mercado, que pode se manifestar em diferentes etapas no processo de produção, comercialização e consumo. Tal fragilidade pode ser apresentada pelo consumidor, bem como por trabalhadores envolvidos na produção e comercialização do bem e/ou indiretamente por outros indivíduos e pela sociedade em geral, em virtude de danos que os processos mercadológicos podem causar ao meio ambiente natural, social e cultural. As experiências de vulnerabilidade podem ser mais prováveis e/ou mais graves em indivíduos e grupos com algum tipo de limitação.

Nossa proposição avança com relação às anteriores por reconhecer a vulnerabilidade em todo o sistema de marketing e não apenas no consumidor final do produto. Os sistemas de marketing podem ser definidos como "[...] redes de indivíduos, grupos ou entidades ligadas direta ou indiretamente através de participação em processo de troca que criam, montam, transformam e tornam disponíveis produtos, tangíveis ou intangíveis em resposta às demandas dos consumidores" (Layton, 2007, p. 230). Consideramos que olhar o mercado pela ideia de sistema de marketing é importante para compreender que a vulnerabilidade é muito mais provável e corrente do que simplesmente olhar as práticas não éticas de algumas empresas. 
Entendemos ser fundamental que a Administração e, mais especificamente, Marketing e Estudos de Consumo interessem-se por conhecimento que proteja e beneficie o consumidor e a sociedade em geral. Hunter-Jones, Baron e Warnaby (2014, p. 107) argumentam que "[...] a falta de pesquisa até o momento é uma tentativa real de diferenciar as necessidades daqueles que experimentam vulnerabilidades temporárias ou permanentes" e, embora existam progressos, "[...] permanece o compromisso fundamental entre proteger os consumidores em risco e não rotular ou marginalizar segmentos de consumidores" (Rittenburg \& Lunde, 2016, p. 190). Por isso, este artigo é uma chamada para a ação, para que os mercados sejam investigados pela vulnerabilidade dos consumidores, dos trabalhadores envolvidos na produção e comercialização de ofertas no mercado, bem como do meio ambiente. 


\section{REFERÊNCIAS}

Adkins, N. R., \& Jae, H. (2010). Marketplace vulnerability of limited English proficient consumers: opportunities to increase knowledge in macromarketing. Journal of Macromarketing, 30(1), 93-104.

Adkins, N. R., \& Ozanne, J. L. (2005). The low literate consumer. Journal of Consumer Research, 32(1),93-105.

Arnould, E. J., \& Thompson, C. J. (2005). Consumer culture theory (CCT): twenty years of research. Journal of Consumer Research, 31(4), 868-882.

Bagozzi, R. P. (1977). Structural equation models in experimental research. Journal of Marketing Research, 14(2), 209-226.

Baker, S. M. (2009). Vulnerability and resilience in natural disasters: a marketing and public policy perspective. Journal of Public Policy \& Marketing, 28(1), 114-123.

Baker, S. M., Geiger-Oneto, S., \& Gentry, J. W. (2011). Potential vs. actual vulnerability in the marketplace. In Proceedings of 36 Annual Macromarketing Conference, Laramie, Wyoming. Recuperado de http://society.macromarketing.org/conference

Baker, S. M., Gentry, J. W., \& Rittenburg, T. L. (2005). Building understanding of the domain of consumer vulnerability. Journal of Macromarketing, 25(2), 128-139.

Baker, S. M., \& Mason, M. (2012). Toward a process theory of consumer vulnerability and resilience: Illuminating its transformative potential. In D. G. Mick (Ed.), Transformative consumer research for personal and collective well-being (pp. 571-592). New York, NY: Routledge.

Barros, D. F., Merabet, D. D. O. B., \& Gouveia, T. M. A. (2015). Público-alvo ou consumidor vulnerável? Histórico da legislação sobre a publicidade infantil em uma perspectiva de macromarketing. In Anais do 6 o Congresso Nacional de Administração e Contabilidade, Rio de Janeiro, RJ.

Berg, L. (2015). Consumer vulnerability: are older people more vulnerable as consumers than others? International Journal of Consumer Studies, 39(4), 284-293.

Bitencourt, J. O. D. S. (2004). O princípio da vulnerabilidade: fundamento da proteção jurídica do consumidor. Revista da EMERJ, 7(25), 248-265.

Boschi, M. R., Barros, D. F., \& Sauerbronn, J. F. R. (2016). A introdução da disciplina de marketing no Brasil: "uma linguagem comum que nos une". Farol-Revista de Estudos Organizacionais e Sociedade, 3(8), 888-956

Brenkert, G. G. (1998). Marketing and the vulnerable. Business Ethics Quarterly, 1(spe.), 7-20.

Caplovitz, D. (1963). Poor pay more: consumer practices of low-income families. New York, NY: Free Press.

Coda, R. C., \& Castro, G. H. C. D. (2019). Marketing business-tobusiness: análise da produção científica brasileira de 2008 a 2018. Revista de Administração de Empresas, 59(4), 258-270.

Commuri, S., \& Ekici, A. (2008). An enlargement of the notion of consumer vulnerability. Journal of Macromarketing, 28(2), 183-186.

Demo, G., Fogaça, N., \& Costa, A. C. (2018). Políticas e práticas de gestão de pessoas nas organizações: cenário da produção nacional de primeira linha e agenda de pesquisa. Cadernos EBAPE.BR, 16(2), 250-263.

Diniz, I. S. F. N., Pereira, R. D. C. D. F., \& Bellini, C. G. P. (2014). Limitações digitais em adolescentes: um estudo sob a perspectiva da teoria da vulnerabilidade do consumidor. In Anais do 6o Encontro de Marketing da Associação Nacional de Pós-Graduação e Pesquisa em Administração, Porto Alegre, RS.

Ertekin, Z. O., \& Atik, D. (2015). Aging of youth through fashion: influential dynamics and societal implications. In Proceedings of 40 음 Annual Macromarketing Conference, Laramie, Wyoming. Recuperado de http://society.macromarketing.org/conference/

Ferenhof, H. A., \& Fernandes, R. F. (2016). Desmistificando a revisão de literatura como base para redação científica: método SSF. Revista $A C B, 21(3), 550-563$.

Ferrari, A., \& Takey, D. G. (2014). O princípio da vulnerabilidade no código de defesa do consumidor. JICEX, 3(3), 1-7.

Gould, S., \& Semaan, R. W. (2014). Avoiding throwing out the baby with the bathwater: critically deconstructing contested positions on social and macromarketing in the health domain. Journal of Macromarketing, 34(4), 520-531.

Hackley, C. (2009). Parallel universes and disciplinary space: the bifurcation of managerialism and social science in marketing studies. Journal of Marketing Management, 25(7-8), 643-659.

Hamilton, K., Dunnett, S., \& Piacentini, M. (2015). Consumer vulnerability: Conditions, contexts and characteristics. London, UK: Routledge.

Harrison, P., \& Massi, M. (2011). Congratulations, you're pre-approved: the policy implications of credit limit upselling. In Proceedings of 36o Annual Macromarketing Conference, Laramie, Wyoming. Recuperado de http://society.macromarketing.org/conference/

Hemais, M. W., \& Faria, A. D. A. (2012). Um olhar sobre consumerismo que marketing pouco atenta (um tipo de miopia?). In Anais do 36응 Encontro Nacional da Associação Nacional de Pós-Graduação e Pesquisa em Administração, Rio de Janeiro, RJ.

Hilhorst, D., \& Bankoff, G. (2004). Introduction: mapping vulnerability. In G. Bankoff, G. Frerks, \& D. Hilhorst (Eds.), Mapping vulnerability: Disasters, Development and People. London, UK: Earthscan.

Hill, R. P. (2005). Special Issue on Vulnerable Consumers. Journal of Macromarketing, 2(2), 201.

Hunt, S. D. (1976). The nature and scope of marketing. Journal of Marketing, 40(3), 17-28.

Hunt, S. D., \& Burnett, J. J. (1982). The macromarketing/micromarketing dichotomy: a taxonomical model. Journal of Marketing, 46(3), 11-26.

Hunter-Jones, P., Baron, S., \& Warnaby, G. (2014). Exploring the Temporality of Consumer Vulnerability. In Proceedings of 39ㅇ Annual Macromarketing Conference, Laramie, Wyoming. Recuperado de http://society.macromarketing.org/conference/

Jones, J. L., \& Middleton, K. L. (2007). Ethical decision-making by consumers: the roles of product harm and consumer vulnerability. Journal of Business Ethics, 70(3), 247-264. 
Karpatkin, R. H. (1999). Toward a fair and just marketplace for all consumers: the responsibilities of marketing professionals. Journal of Public Policy \& Marketing, 18(1), 118-122.

Kuzma, E. L., Doliveira, S. L. D., \& Silva, A. Q. (2017). Competências para a sustentabilidade organizacional: uma revisão sistemática. Cadernos EBAPE.BR, 15(esp.), 428-444.

Layton, R. A. (2007). Marketing systems - a core macromarketing concept. Journal of Macromarketing, 27(3), 227-242.

Layton, R. A., \& Grossbart, S. (2006). Macromarketing: past, present, and possible future. Journal of Macromarketing, 26(2), 193-213.

Lei no 8.078, de 11 de setembro de 1990. (1990). Dispõe sobre a proteção do consumidor e dá outras providências. Recuperado de http://www.planalto.gov.br/ccivil_03/leis/L8078.htm

Lima, S. M. D. (2011). Vulnerabilidade e Hipossuficiência na sistemática do Código de Defesa do Consumidor. Revista do Centro Acadêmico Afonso Pena, 14(2), 241-259.

Macromarketing Conference. (2018). In Proceedings of 43으슨 Macromarketing Conference, Laramie, Wyoming. Recuperado de http://society.macromarketing.org/conference/

Mansfield, P. M., \& Pinto, M. B. (2008). Consumer vulnerability and credit card knowledge among developmentally disabled citizens. Journal of Consumer Affairs, 42(3), 425-438.

Mason, M., \& Pavia, T. (2014). Health and consumer vulnerability: identity dissolution and resiliency behaviors. In J. Cotte, \& S. Wood (Eds.), NA - Advances in Consumer Research (v. 42, pp. 7-11). Duluth, MN: Association for Consumer Research.

McKeage, K. K., Crosby, E., \& Rittenburg, T. L. (2015). Gender identity and consumer vulnerability. In Proceedings of 40 o Annual Macromarketing Conference, Laramie, Wyoming. Recuperado de http://society.macromarketing.org/conference/

Miranda, C. M. C., \& Arruda, D. M. D. O. (2004). A evolução do pensamento de marketing: uma análise do corpo doutrinário acumulado no século XX. Revista Interdisciplinar de Marketing, 3(1), 40-57.

Mirosa, M., \& Wooliscroft, B. (2013). Experiencing vulnerability “everyday": Food choice. In Proceedings of 38ㅇ Annual Macromarketing Conference, Laramie, Wyoming. Recuperado de http://society.macromarketing. org/assets/proceedings/2013-macromarketing-proceedings.pdf

Mittelstaedt, J. D., Duke, C. R., \& Mittelstaedt, R. A. (2009). Health care choices in the United States and the constrained consumer: a marketing systems perspective on access and assortment in health care. Journal of Public Policy \& Marketing, 28(1), 95-101.

Nason, R. W. (1989). The social consequences of marketing: macromarketing and public policy. Journal of Public Policy \& Marketing, $8(1), 242-251$.

Nason, R. W. (2011). Institutionalization of macromarketing. Journal of Historical Research in Marketing, 3(2), 261-268.

Oliveira, E. Q. D., Jr. (2010). O conceito de vulnerabilidade no direito penal. Recuperado de https://lfg.jusbrasil.com.br/noticias/2146510/o-conceitode-vulnerabilidade-no-direito-penal-eudes-quintino-de-oliveira-junior

Pavia, T. M., \& Mason, M. J. (2014). Vulnerability and physical, cognitive, and behavioral impairment: Model extensions and open questions. Journal of Macromarketing, 34(4), 471-485.
Pearson, Y. E., \& Liu-Thompkins, Y. (2012). Consuming direct-toconsumer genetic tests: the role of genetic literacy and knowledge calibration. Journal of Public Policy \& Marketing, 31(1), 42-572.

Pechmann, C. C., Levine, L., Loughlin, S., \& Leslie, F. (2005). Impulsive and self-conscious: adolescents vulnerability to advertising and promotion. Journal of Public Policy \& Marketing, 24(2), 202-221.

Pechmann, C. C., Moore, E. S., Andreasen, A. R., Connell, P. M., Freeman, D., Gardner, M. P. ... \& Soster, R. L. (2011). Navigating the central tensions in research on at-risk consumers: challenges and opportunities. Journal of Public Policy \& Marketing, 30(1), 23-30.

Piacentini, M., Dunnett, S., \& Hamilton, K. (2014). Patient, client, user, consumer? Issues involved with approaching vulnerability with consumer-focused terminology. In Proceedings of 38 Annual Macromarketing Conference, Laramie, Wyoming. Recuperado de http://society.macromarketing.org/conference/

Pinto, M. R., Batinga, G. L., Ássimos, B. M., \& Almeida, B. T. (2016). Transformative consumer research (TCR): reflexões, diretrizes e uma análise do campo no Brasil. Revista Interdisciplinar de Marketing, 6(2), 54-66.

Rittenburg, T. L., \& Lunde, M. B. (2016). Ethics in target market selection: A historical perspective. In Proceedings of 410 Annual Macromarketing Conference, Laramie, Wyoming. Recuperado de http://society.macromarketing.org/conference/

Santo, P. O. D. E., \& Hemais, M. W. (2017). Discriminação contra consumidores de baixa renda. Revista Pretexto, 18(1), 64-79.

Sauerbronn, J. F. R. (2013). Diga-me o que publicas e te direi quem és: perspectivas e resistências na publicação da produção de conhecimento em Marketing. Revista de Negócios, 18(1), 42-52.

Shapiro, S. J., Tadajewski, M., \& Shultz II, C. J. (2009). Interpreting macromarketing: the construction of a major macromarketing research collection. Journal of Macromarketing, 29(3), 325-334.

Sheth, J. N., \& Sisodia, R. S. A dangerous divergence: marketing and society. Journal of Public Policy \& Marketing, 24(1), 160-162.

Shultz II, C. J., \& Holbrook, M. B. (2009). The paradoxical relationships between marketing and vulnerability. Journal of Public Policy \& Marketing, 28(1), 124-127.

Silva, J. O. D., Abreu, N. R. D., \& Mano, R. F. (2015). Consumidores vulneráveis ou vulnerabilizados? Uma reflexão sobre a acessibilidade em meios hoteleiros na ótica das pessoas com deficiência física. In Anais do 29o Encontro Nacional da Associação Nacional de PósGraduação e Pesquisa em Administração, Belo Horizonte, MG.

Silva, R. O. D., \& Barros, D. F. (2018). A Vulnerabilidade do consumidor no mercado do morrer. In Anais do 8 Encontro de Marketing da Associação Nacional de Pós-Graduação e Pesquisa em Administração, Porto Alegre, RS.

Silva, R. O. D., Barros, D. F., \& Gouveia, T. M. D. O. A. (2017). Eu tenho a força! A popularização do consumo de suplementos alimentares e a vulnerabilidade do consumidor. Revista ADM.MADE, 21(1), 34-50.

Smith, N. C., \& Cooper-Martin, E. (1997). Ethics and target marketing: the role of product harm and consumer vulnerability. Journal of Marketing, 61(3), 1-20. 
Uma discussão necessária sobre a vulnerabilidade do consumidor: avanços, lacunas e novas perspectivas
Rosana Oliveira da Silva | Denise Franca Barros

Tânia Maria de Oliveira Almeida Gouveia Daniel de Oliveira Barata Merabet
Souza, A. D. S., \& Silva, C. P. D. (2006). O consumo na vida de adolescentes de diferentes condições socioeconômicas: uma reflexão para o marketing no Brasil. Cadernos EBAPE.BR, 4(1), 1-18.

Tipton, M. M., Bharadwaj, S. G., \& Robertson, D. C. (2009). Regulatory exposure of deceptive marketing and its impact on firm value. Journal of Marketing, 73(6), 227-243.

Visconti, L. M. (2016). A conversational approach to consumer vulnerability: performativity, representations, and storytelling. Journal of Marketing Management, 32(3-4), 371-385.

Viswanathan, M., \& Gau, R. (2005). Functional illiteracy and nutritional education in the United States: a research-based approach to the development of nutritional education materials for functionally illiterate consumers. Journal of Macromarketing, 25(2), 187-201.

Wilkie, W. L., \& Moore, E. S. (2003). Scholarly research in marketing: exploring the " 4 eras" of thought development. Journal of Public Policy \& Marketing, 22(2), 116-146.

Wolburg, J. M. (2005). Drawing the line between targeting and patronizing: how "vulnerable" are the vulnerable?. Journal of Consumer Marketing, 22(5), 287-288.

Woodliffe, L. (2007). An empirical re-evaluation of consumer disadvantage. International Review of Retail, Distribution and Consumer Research, 17(1), 1-21.

Rosana Oliveira da Silva

ORCID: https://orcid.org/0000-0003-3971-5217

Doutoranda em Administração na Universidade do Grande Rio (UNIGRANRIO); Servidora da Fundação Oswaldo Cruz (FIOCRUZ).

E-mail: rooliveira35@outlook.com

Denise Franca Barros

ORCID: https://orcid.org/0000-0003-1640-6171

Doutora em Administração pela Fundação Getulio Vargas (FGV); Professora do Programa de Pós-Graduação em Administração da Universidade do Grande Rio (PPGA-UNIGRANRIO).E-mail: denise.barros@unigranrio.edu.br

Tânia Maria de Oliveira Almeida Gouveia ORCID: https://orcid.org/0000-0002-8231-9714

Doutora em Administração pela Fundação Getulio Vargas (FGV); Professora da Universidade do Estado do Rio de Janeiro (UERJ).

E-mail: almeida.tania@globo.com

Daniel de Oliveira Barata Merabet

ORCID: https://orcid.org/0000-0003-4582-3898

Doutor em Administração pela Universidade do Grande Rio (UNIGRANRIO). E-mail: danmerabet@gmail.com 